\title{
Charged Current Universality in the Minimal Supersymmetric Standard Model
}

\author{
A. Kurylov ${ }^{1,2}$ and M. J. Ramsey-Musolf ${ }^{1,2}$ \\ ${ }^{1}$ Kellogg Radiation Laboratory, California Institute of Technology, Pasadena, California 91125 \\ ${ }^{2}$ Department of Physics, University of Connecticut, Storrs, Connecticut 06269
}

(Received 24 September 2001; published 1 February 2002)

\begin{abstract}
We compute the complete one-loop contributions to low-energy charged current weak interaction observables in the minimal supersymmetric standard model (MSSM). We obtain the constraints on the MSSM parameter space which arise when precision low-energy charged current data are analyzed in tandem with measurements of the muon anomaly. While the data allow the presence of at least one light neutralino, they also imply a pattern of mass splittings among first and second generation sleptons and squarks which contradicts predictions of widely used models for supersymmetry-breaking mediation.
\end{abstract}

DOI: $10.1103 /$ PhysRevLett.88.071804

The universality of the charged current weak interaction (CCWI) is an important feature of the standard model $(\mathrm{SM})$. The presence of a common coupling strength and $(V-A) \times(V-A)$ current-current interaction structure for all CCWI processes has been tested with high precision in a number of leptonic and semileptonic experiments. The results place significant limits on scenarios for physics beyond the SM which may generate breakdowns of CCWI universality. To date, however, the implications of universality tests for supersymmetric extensions of the SM - a leading candidate for "new physics"- have not been fully explored. Although supersymmetric theories which break $R$-parity conservation have been considered [1-3], no analysis has been performed for the simplest version of supersymmetry (SUSY): the minimal supersymmetric standard model (MSSM). In this Letter, we report on results obtained from such an analysis.

Low-energy SUSY is an attractive scenario from a number of standpoints: it provides a solution to the hierarchy problem associated with Higgs mass renormalization, it produces coupling unification at the grand-unified-theory (GUT) scale, and it is a prediction of superstring theory. It remains to be seen, however, which version of SUSY correctly describes electroweak phenomena, and, as we argue below, low-energy precision measurements may provide important clues. In particular, details of the superpartner spectrum (e.g., masses and mixing angles) are largely unknown. Limits on branching ratios obtained from collider data provide, in general, only weak lower bounds. Low-energy CCWI observables can provide complementary information, since they effectively compare the relative importance of effects produced by different species or generations of superpartners.

In the MSSM, $R$ parity (and, thus, $B-L$ ) is conserved, so that SUSY corrections to low-energy observables arise only via tiny loop effects (nonconservation of $R$ parity allows for the presence of new tree-level SUSY effects). In order to become sensitive to such contributions, one generally requires a precision of $\sim(\alpha / \pi) \times(M / \tilde{M})^{2}$, where $M$ is the relevant mass of a SM particle and $\tilde{M}$ is a superpartner mass. The recent report [4] of a $2.6 \sigma$ deviation
PACS numbers: 12.60.Jv, 12.15.Ji, 12.15.Lk, 13.40.Em

of the muon anomaly from the SM prediction provides the first, tantalizing hint of MSSM loop effects. In this case, $M=m_{\mu}$, making the part per million precision of the muon anomaly measurement sensitive to superpartner masses on the order of a few hundred $\mathrm{GeV}$ [5].

In contrast, for CCWI observables, one has $M \sim M_{W}$, so that only a few $\times 10^{-3}$ precision is needed to achieve comparable sensitivity. A number of lowenergy CCWI measurements have achieved this level of precision, and others are poised to do so in the near future. These observables include (a) the branching ratio $R_{e / \mu}=\Gamma\left(\pi^{+} \rightarrow e^{+} \nu_{e}+\pi^{+} \rightarrow e^{+} \nu_{e} \gamma\right) / \Gamma\left(\pi^{+} \rightarrow\right.$ $\mu^{+} \nu_{\mu}+\pi^{+} \rightarrow \mu^{+} \nu_{\mu} \gamma$ ), (b) the $f t$ values for "superallowed" $\left(J^{\pi}, I\right)=\left(0^{+}, 0\right) \rightarrow\left(0^{+}, 0\right)$ Fermi nuclear $\beta$-decays, and (c) the neutron lifetime, $\tau_{n}$. In addition, a more precise determination of $\tau_{n}$ is underway at NIST, as are measurements of the parity-violating neutron $\beta$-decay parameter $A$ at LANSCE and the $\pi^{+} \beta$-decay branching ratio at PSI.

Of particular interest for our analysis are the results of superallowed nuclear $\beta$ decays, from which one extracts the Cabibbo-Kobayashi-Maskawa (CKM) quark mixing matrix element $\left|V_{u d}\right|$. When $\left|V_{u d}\right|$ is considered along with the values of $\left|V_{u s}\right|$ and $\left|V_{u b}\right|$ determined from $K_{e 3}$ and $B$ meson decays, respectively, one obtains for the sum of the squares a result falling below the unitarity requirement by $2.2 \sigma$ [6]. In what follows, we discuss the implications of this deviation - which will be tested via the new $\tau_{n}$ and A measurements - for the MSSM spectrum.

Before considering MSSM effects in detail, it is useful to review the general features of nonuniversal CCWI contributions. Any CCWI amplitude is properly normalized to $G_{\mu}$, the Fermi constant measured in $\mu$ decay-being one of the three most precise inputs for the gauge sector of the MSSM. It is related to the universal weak coupling $g$ and the $W$-boson mass as

$$
\frac{G_{\mu}}{\sqrt{2}}=\frac{g^{2}}{8 M_{W}^{2}}\left[1+\Delta r_{\mu}\right],
$$

where $\Delta r_{\mu}$ includes the effects of weak, radiative corrections in the MSSM as well as other possible new physics 
contributions to $\mu$ decay. The MSSM amplitudes for other CCWI processes are $\propto g^{2} / M_{W}^{2}$, and, in order to express them in terms of $G_{\mu}$, one must invert relation (1). For example, the Fermi constant relevant for light-quark $\beta$ decay has the form

$$
G_{F}^{\beta}=G_{\mu} V_{u d}\left(1-\Delta r_{\mu}+\Delta r_{\beta}\right),
$$

where $\Delta r_{\beta}$ contains the MSSM electroweak radiative corrections and other possible new physics contributions to the semileptonic decay amplitude.

The difference $\Delta r_{\beta}-\Delta r_{\mu}$ is sensitive to nonuniversal effects. Modifications of the $W$-boson propagator, which are universal, cancel from this difference, leaving only sensitivity to non-universal vertex corrections, box diagrams, and external leg corrections. We have computed these corrections in the MSSM, where-owing to $R$-parity conservation - the loops always contain an even number of superpartners: $\operatorname{spin}-0$ sfermions $(\tilde{f})$, spin- $1 / 2$ gluinos $(\tilde{g})$, and spin-1/2 mixtures of electroweak gauginos and Higgsinos - the neutralinos $\left(\tilde{\chi}_{1-4}^{0}\right)$ and charginos $\left(\tilde{\chi}_{1,2}^{+}\right)$. Because it compares these corrections as they appear in leptonic and semileptonic decay amplitudes, $G_{F}^{\beta}$ is essentially a measure of slepton-squark universality in the MSSM. Moreover, since both $\Delta r_{\beta}$ and $\Delta r_{\mu}$ pertain to processes with $e^{+} \nu_{e}\left(e^{-} \bar{\nu}_{e}\right)$ in the final state, the difference $\Delta r_{\beta}-\Delta r_{\mu}$ is considerably more sensitive to effects produced by second generation sleptons than to those produced by the first generation. In a similar way, the ratio $R_{e / \mu}$ compares different leptonic final states for a given hadronic initial state, making it effectively a probe of $\tilde{e}-\tilde{\mu}$ universality. At present, the experimental error bars in $R_{e / \mu}$ are roughly a factor of 2 larger than necessary to produce significant constraints, so we focus on the more restrictive implications of light-quark $\beta$-decay data.

In the limit of unbroken SUSY, the parameters of the MSSM are those of the SM, modulo a few consequences of supersymmetry: the MSSM Lagrangian contains two Higgs doublets $\left(H_{u}, H_{d}\right)$ and one new dimensionful parameter $(\mu)$ as compared to the SM [7]. In order to break SUSY, thereby splitting the masses of the SM particles from their superpartners, one must introduce a "soft" SUSY-breaking Lagrangian, $\mathcal{L}_{\text {soft }}$, whose dimensionful parameters are at most a few $\times$ the weak scale. As a result, the MSSM contains 105 new parameters not present in the SM. A number of scenarios have been proposed for simplifying $\mathcal{L}_{\text {soft }}$ into a more fundamental theory [8]. The task for phenomenology is to determine which of these proposals is most consistent with the data and which additional measurements could provide new constraints on soft SUSY-breaking.

For this purpose, it is useful to identify the independent parameters which must be determined. In the electroweak gauge and Higgs sectors, one has the couplings $g$ and $g^{\prime}$, the vacuum expectation values of the Higgs $v_{u}$ and $v_{d}$, the SUSY mass parameter $\mu$, along with the SUSY-breaking Higgs mass parameters, $m_{H_{u}}^{2}, m_{H_{d}}^{2}, b$ and gaugino masses,
$M_{1}$ and $M_{2}$. In general, $g, g^{\prime}$, and $v=\sqrt{v_{u}^{2}+v_{d}^{2}}$ can be determined from $\alpha, G_{\mu}$, and $M_{Z}$, while $|\mu|$ and $b$ can be expressed in terms of $M_{Z}, m_{H_{u}}^{2}, m_{H_{d}}^{2}$ and $\tan \beta=$ $v_{u} / v_{d}$. The strong gauge sector of the theory contains the $\mathrm{SU}(3)_{c}$ gauge coupling, $C P$-violating $\theta$ parameter, and gluino mass, $M_{3}$. Inclusion of fermions and their superpartners introduces the SUSY Yukawa couplings to the Higgs fields as well as SUSY-breaking quadratic sfermion mass terms and triboson Higgs-sfermion-sfermion interactions. As a consequence of these interactions, left- and right-handed sfermions $\left(\tilde{f}_{L, R}\right)$ can mix, much as mass terms in the SM Lagrangian lead to mixing between ordinary fermions. The sine of the $L-R$ mixing angle $\theta_{L R}$ is proportional to the off-diagonal element of the sfermion mass-squared matrix:

$$
M_{L R}^{2}=\left\{\begin{array}{ll}
m_{f}\left(\mu \tan \beta-A_{f}\right), & q_{f}<0 \\
m_{f}\left(\mu \cot \beta-A_{f}\right), & q_{f}>0
\end{array},\right.
$$

where $m_{f}$ and $q_{f}$ are the fermion mass and charge, respectively, and $A_{f}$ is the SUSY-breaking triboson coupling, normalized to $m_{f}$ as in minimal supergravity (mSUGRA) models.

To demonstrate the impact of CCWI observables on the MSSM parameter space, it is useful to distinguish contributions to the corrections $\Delta r^{\mathrm{MSSM}}$ arising from the $\mathrm{SM}$ particles $\left(\Delta r^{\mathrm{SM}}\right)$ and their superpartners $\left(\Delta r^{\mathrm{SUSY}}\right)$, where the former include contributions from the two Higgs doublets. In computing $\Delta r^{\mathrm{MSSM}}$, we follow the standard practice of neglecting terms quadratic in light lepton and quark masses. To avoid large flavor-changing neutral currents, we also assume the same pattern of generation mixing among sfermions as for the SM fermions. In treating strong interaction contributions to the semileptonic amplitudes, we adopt the framework of an effective field theory: short distance effects ( $p>\Lambda, \Lambda \sim 1 \mathrm{GeV}$ ) are included explicitly in renormalized operators while long distance $(p<\Lambda)$, nonperturbative effects are contained in $\Lambda$-dependent matrix elements. The latter have been studied extensively elsewhere [6,9], and we do not discuss them here.

We begin our analysis of the parameter space by taking each superpartner species $\left(\tilde{\chi}, \tilde{\mu}, \tilde{\nu}_{\mu}, \tilde{u}, \tilde{d}, \tilde{g}\right)$ to have at least one light member whose mass is given by the collider lower bound [10]. In addition, we assume identical masses and mixing angles for $\tilde{u}$ and $\tilde{d}$ ("squark universality"). Doing so suppresses gluino loop corrections to the hadronic vector current; we analyze these effects separately below. Under these assumptions, the nuclear $G_{F}^{\beta}$ determinations favor maximal mixing among sfermions, with larger mass splittings between smuons than between squarks. To illustrate, we plot in Fig. 1 the CCWI constraints on mass ratios $\kappa_{\tilde{f}} \equiv M_{\tilde{f}_{2}} / M_{\tilde{f}_{1}}$ for smuons and squarks. The constraints assume maximal mixing, which occurs for $M_{L R}^{2} \gg\left|M_{L}^{2}-M_{R}^{2}\right|$, where $M_{L, R}^{2}$ are the diagonal mass-squared matrix entries. The dashed line indicates the constraints obtained from $G_{F}^{\beta}$, where we have 


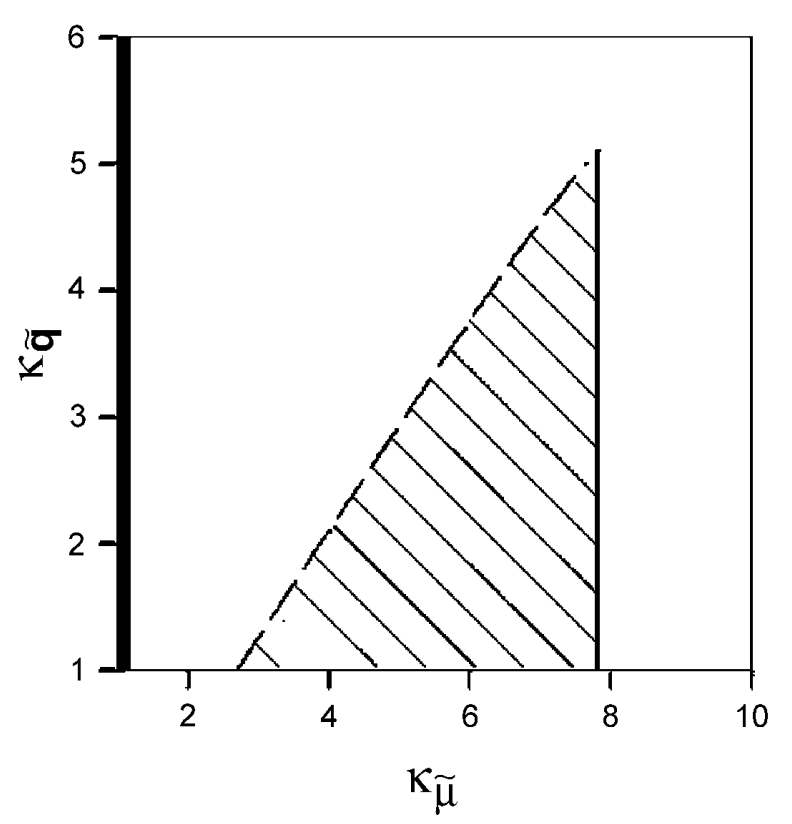

FIG. 1. Slepton-squark universality constraints $(2 \sigma)$ for "universal" first generation squarks with maximal mixing. Shading indicates region allowed by $G_{F}^{\beta}$ (dashed line) and $G_{\mu}-\alpha-M_{Z}-M_{W}$ relation (solid line). Thick vertical bar indicates the $(g-2)_{\mu}$ allowed region $\left(1<\kappa_{\tilde{\mu}} \leq 1.04\right)$.

required that nonuniversal SUSY corrections to this process produce no additional deviation from CKM unitarity. This requirement implies that $\Delta r_{\beta}^{\mathrm{SUSY}}-\Delta r_{\mu}^{\mathrm{SUSY}}<$ 0 (95\% confidence).

The results indicate that the relative splitting between charged smuons must be greater than that among squarks in order to avoid exacerbating the CKM unitarity deviation. This constraint may be understood by considering the asymptotic expression for the nonuniversal corrections, which is dominated by vertex corrections involving $\tilde{f}-\tilde{\chi}^{+}-\tilde{\chi}^{0}$ intermediate states. For large $\kappa_{\tilde{f}}$, one has, for the dominant SUSY contribution,

$$
\Delta r_{\beta}^{\mathrm{SUSY}}-\Delta r_{\mu}^{\mathrm{SUSY}} \sim \alpha \frac{\left(c^{2}-s^{2}\right)}{32 \pi s^{2} c^{2}} \ln \left(\kappa_{\tilde{q}}^{2} / \kappa_{\tilde{\mu}}^{4}\right)+\cdots,
$$

where $c(s)$ denotes the cosine (sine) of the weak mixing angle. In order for this correction to be negative, the squark effect must be smaller than the slepton effect. In particular, for nearly degenerate squarks $\left(\kappa_{\tilde{q}} \sim 1\right)$, one needs $M_{\tilde{\mu}_{2}} \gtrsim$ $3 M_{\tilde{\mu}_{1}}$. Note that the presence of significant $L-R$ mixing obscures the expected $(M / \tilde{M})^{2}$ scaling of the corrections, yielding instead a logarithmic dependence on superpartner mass ratios.

For comparison, we also give the constraints obtained from a comparsion of the experimental value for $G_{\mu}$ with its MSSM prediction using $\alpha, M_{Z}$, and $M_{W}$ as inputs. In using Eq. (1), one has

$$
G_{\mu}^{\mathrm{MSSM}}=\frac{\pi \alpha M_{Z}^{2}}{\sqrt{2} M_{W}^{2}\left(M_{Z}^{2}-M_{W}^{2}\right)\left[1-\Delta r_{\mu}^{\mathrm{MSSM}}\right]} .
$$

Requiring $G_{\mu}^{\mathrm{MSSM}}$ to be consistent with the experimental value implies the $2 \sigma$ constraint [11] $-0.0091 \leq$ $\Delta r_{\mu}^{\mathrm{SUSY}} \leq 0.0037$, where the range is determined primarily by the experimental uncertainties in $M_{W}$ and $m_{t}$ [10]. The correction $\Delta r_{\mu}^{\mathrm{SUSY}}$ samples both nonuniversal corrections as well as universal corrections entering the $W$-boson propagator. The resulting constraints, indicated by the solid line in Fig. 1, lead to upper bounds on the degree of nondegeneracy among sfermions. We observe that the range of allowed mass splittings among sfermions is tightened when the light-quark $\beta$ decay and $W$ mass measurements are combined.

SUSY contributions to the muon anomaly are also sensitive to smuon mixing, with the $\tilde{\mu}-\tilde{\chi}^{0}-\tilde{\chi}^{0}$ vertex correction also being proportional to $M_{L R}^{2}$. (The $\tan \beta$ dependence of the CCWI corrections is governed by its appearance in $M_{L R}^{2}$. Hence, we focus on the latter rather than on $\tan \beta$.) For light $\tilde{\mu}$ and $\tilde{\chi}^{0}$, the recent E821 results imply nearly minimal mixing. Reducing the degree of smuon mixing, however, reduces the region of the $\kappa_{\tilde{\mu}}-\kappa_{\tilde{q}}$ parameter space allowed by the CCWI data and $W$ mass measurements. For nearly minimal mixing as implied by the muon anomaly, there exists no allowed region. Consequently, one must relax the assumptions that each species of superpartner has at least one light member or that up- and down-squark masses and mixing angles are identical. We consider each possibility in turn.

(i) Increase the mass of the lightest $\tilde{\chi}^{0}$ and $\tilde{\chi}^{+}$. Doing so weakens the $(g-2)_{\mu}$ constraints on smuon mixing. For sufficiently heavy $\tilde{\chi}^{0,+}$, however, the effect of the dominant vertex correction to $G_{F}^{\beta}$ is also suppressed, leading to a change of sign in the difference $\Delta r_{\beta}^{\text {SUSY }}-\Delta r_{\mu}^{\text {SUSY }}$ (the contribution from other vertex corrections and box graphs is positive). In order to keep this difference negative, one must increase $\kappa_{\tilde{\mu}}$, leading to a correlation between $\kappa_{\tilde{\mu}}$ and $M_{\tilde{\chi}^{0}}$. For $M_{\tilde{\chi}^{0}}<1 \mathrm{TeV}$, the allowed regions determined by the $(g-2)_{\mu}$ and $G_{F}^{\beta}$ constraints never overlap.

(ii) Increase the mass of the lightest $\tilde{\mu}$ (and, thus, $\tilde{\nu}_{\mu}$ ) for fixed $M_{\tilde{u}, \tilde{d}}$ and $\kappa_{\tilde{\mu}}$. While this parameter variation desensitizes the muon anomaly to smuon mixing, it also reduces the region allowed by the comparison of $G_{\mu}^{\mathrm{MSSM}}$ with the experimental value. When $M_{\tilde{\mu}}$ is sufficiently heavy to evade the $(g-2)_{\mu}$ constraints on smuon mixing, the allowed region of Fig. 1 vanishes. Increasing the mass of the lightest first generation squarks does not affect $(g-2)_{\mu}$, leaving the essential conflict between the muon anomaly and CCWI data (Fig. 1) unresolved.

(iii) Take smuon mixing to be zero, thereby avoiding any $(g-2)_{\mu}$ constraints on $M_{L R}^{2}$. Since the degree of smuon mixing must always be greater than mixing among first generation squarks (Fig. 1), we also take the latter to zero. In this case, the $G_{F}^{\beta}$ constraints lead to a nontrival relationship between the masses of the $\tilde{\mu}_{L}$ and first generation $\tilde{q}_{L}$. In particular, one always has $M_{\tilde{\mu}_{L}}>M_{\tilde{q}_{L}}$ for light $\tilde{\chi}$ and no sfermion mixing. In this case, the dominant MSSM correction to $G_{F}^{\beta}$ has the asymptotic (large $M_{\tilde{f}}$ ) expression 


$$
\Delta r_{\beta}^{\mathrm{SUSY}}-\Delta r_{\mu}^{\mathrm{SUSY}} \sim \frac{\alpha}{2 \pi} \cos 2 \beta\left[\frac{1}{3} \frac{M_{Z}^{2}}{M_{\tilde{q}}^{2}} \ln \frac{M_{\tilde{q}}^{2}}{\left\langle M_{\tilde{\chi}}^{2}\right\rangle}-\frac{M_{Z}^{2}}{M_{\tilde{\mu}}^{2}} \ln \frac{M_{\mu}^{2}}{\left\langle M_{\tilde{\chi}}^{2}\right\rangle}\right]+\ldots
$$

where $\left\langle M_{\tilde{\chi}}^{2}\right\rangle$ is the mass scale associated with the $\tilde{\chi}$ which is much smaller than $M_{\tilde{f}}$ in the asymptotic limit. For $\tan \beta>1$ as favored by lower bounds on the lightest Higgs mass [12], $\cos 2 \beta<0$. To maintain the correct sign for the SUSY contribution to $G_{F}^{\beta}$, one requires $M_{\tilde{\mu}}^{2}>$ $3 M_{\tilde{q}}^{2}$ (up to logarithmic corrections), where the factor of 3 results from the different hypercharges of the doublet left-handed squarks and smuons.

This phenomenological solution is particularly interesting from the standpoint of both gauge-mediated and mSUGRA models of SUSY-breaking mediation, which generally predict $M_{\tilde{q}}>M_{\tilde{\ell}}$. In mSUGRA, this hierarchy results from gluino contributions to the renormalization group running of the masses down from the GUT scale. Inverting this hierarchy would presumably require modifying the universality assumptions made for the parameters of $\mathcal{L}_{\text {soft }}$ at the GUT scale.

(iv) Relax the assumption of squark universality. For illustrative purposes, we take no mixing among $\tilde{u}_{L, R}$ but maximal mixing among $\tilde{d}_{L, R}$ and $\tilde{\mu}_{L, R}$ (similar qualitative conclusions arise if mixing occurs among $\tilde{u}_{L, R}$ instead of $\left.\tilde{d}_{L, R}\right)$. In this case, SUSY $S U(3)_{c}$ corrections to the light-quark current operator dominate $\Delta r_{\beta}^{\mathrm{SUSY}}$ for light gluinos. The resulting $\kappa_{\tilde{\mu}}-\kappa_{\tilde{d}}$ constraints are shown in Fig. 2. The solution in this instance requires significant mixing and mass-splittings among the $d$-type squarks. For $\kappa_{\tilde{\mu}} \sim 1$ as required by $(g-2)_{\mu}$, we require $3 \lesssim$ $\kappa_{\tilde{d}} \lesssim 4.5$. Decreasing the degree of $\tilde{d}_{L, R}$ mixing reduces this range. For $M_{\tilde{d}_{1}} \sim 115 \mathrm{GeV}$, the lower bound of this range implies $M_{\tilde{d}_{2}} \sim 450 \mathrm{GeV}$, or $M_{L R}^{2} / m_{d}=(\mu \tan \beta-$ $\left.A_{d}\right) \sim 10^{7} \mathrm{GeV}$. Increasing the mass of the lightest $\tilde{d}$ (see, e.g., [13]) leads to a corresponding increase in this scale. Short of any miraculous fine-tuning of parameters involving smuons, the $(g-2)_{\mu}$ results cannot accommo-

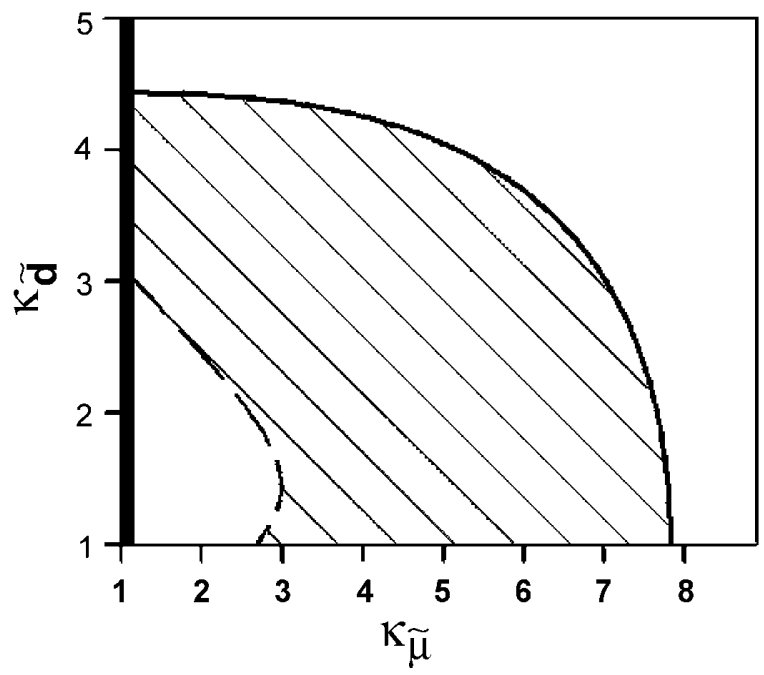

FIG. 2. Same as Fig. 1 but for squark nonuniversality. date values of $\mu \tan \beta$ having this magnitude, so the entire effect would have to arise from $A_{d}$. In models such as mSUGRA, the triboson couplings $A_{f}$ have a common value at the GUT scale. Lower bounds on the mass of the lightest Higgs imply that $\left|A_{t}\right| \lesssim$ a few $\mathrm{TeV}$ at the weak scale [14], while perturbative running of the $A_{f}$ down from a common value at the GUT scale cannot produce a factor of $10^{4}$ difference between $A_{d}$ and $A_{t}$ at the weak scale. Thus, it appears that this solution cannot be accommodated within SUSY-breaking mediation models assuming coupling universality at the GUT scale.

The implications of solution (iv) may be evaded for sufficiently heavy gluinos $\left(M_{\tilde{g}} \gtrsim 500 \mathrm{GeV}\right)$. In this case, however, one returns to the situation characterized by Fig. 1, leaving solution (iii) - which is also inconsistent with the universality of soft SUSY-breaking interactions - as the only viable option.

Alternatively, one may avoid either of these solutions by taking all superpartners to be sufficiently heavy $(\tilde{M} \gtrsim$ $500 \mathrm{GeV})$, a choice disfavored by CCWI and $(g-2)_{\mu}$ data. Indeed, while MSSM loop effects can never completely correct the apparent CKM unitarity violation suggested by $G_{F}^{\beta}$, choosing MSSM parameters near the edges of the allowed regions (for light superpartners) can reduce the discrepancy by $\sim 0.5 \sigma$. More generally, requiring consistency between the MSSM and both CCWI and $(g-2)_{\mu}$ data suggests the necessity for modifying widely used models of SUSY-breaking mediation.

We thank J. Erler, B. Filippone, S. Heinemeyer, John $\mathrm{Ng}$, and P. Vogel for several useful discussions. This work was supported by the Department of Energy and National Science Foundation.

[1] V. Barger and K. Cheung, Phys. Lett. B 480, 149 (2000).

[2] M. J. Ramsey-Musolf, Phys. Rev. D 62, 056009 (2000).

[3] B. C. Allanach, A. Dedes, and H. Dreiner, Phys. Rev. D 60, 075014 (1999).

[4] Muon g-2 Collaboration, H. N. Brown et al., Phys. Rev. Lett. 86, 2227 (2001).

[5] For a recent discussion and reference list, see L. Everett et al., Phys. Rev. Lett. 86, 3484 (2001).

[6] I. S. Towner and J. C. Hardy, nucl-th/9809087.

[7] H. E. Haber and G. L. Kane, Phys. Rep. 117, 75 (1985).

[8] For a review, see Perspectives on Supersymmetry, edited by G. L. Kane (World Scientific, Singapore, 1998).

[9] N. Kaiser, Phys. Rev. C 64, 028201 (2001).

[10] Particle Data Group, http://pdg.lbl.gov/2001

[11] W. Marciano, Phys. Rev. D 60, 093006 (1999).

[12] S. Heinemeyer and G. Weiglein, Nucl. Phys. (Proc. Suppl.) B89, 216 (2000).

[13] J. Erler and D. M. Pierce, Nucl. Phys. B 526, 53 (1998).

[14] S. Heinemeyer, W. Hollik, and G. Weiglein, Phys. Lett. B 455, 179 (1999). 